\title{
Enhanced recovery after pulmonary surgery
}

\author{
Jules Eustache ${ }^{1}$, Lorenzo E. Ferri ${ }^{2}$, Liane S. Feldman ${ }^{3}$, Lawrence Lee ${ }^{3}$, Jonathan D. Spicer ${ }^{2}$ \\ ${ }^{1}$ Department of General Surgery, ${ }^{2}$ Division of Thoracic Surgery, ${ }^{3}$ Steinberg-Bernstein Centre for Minimally Invasive Surgery and Innovation, \\ McGill University Health Centre, Montreal, Canada \\ Contributions: (I) Conception and design: All authors; (II) Administrative support: J Eustache, JD Spicer, LS Feldman; (III) Provision of study \\ materials or patients: J Eustache, JD Spicer, LS Feldman; (IV) Collection and assembly of data: None; (V) Data analysis and interpretation: None; (VI) \\ Manuscript writing: All authors; (VII) Final approval of manuscript: All authors. \\ Correspondence to: Dr. Jonathan D. Spicer, MD, PhD. Division of Thoracic Surgery, McGill University Health Centre, Montreal, Canada. \\ Email: jonathan.spicer@mcgill.ca.
}

\begin{abstract}
The concept of surgical recovery encompasses the entire perioperative phase of the patient, beginning with the preoperative baseline and culminating in the long-term rehabilitation of the patient in the post-operative phase. Enhanced recovery pathways (ERPs) aim to encompass all phase of the patient trajectory, including the preoperative, perioperative, and postoperative management of surgical patients. While significant literature exists on standardizing and optimizing the perioperative phase, standardizing the pre and post-operative phases remains a topic of debate. Furthermore, with regards to pulmonary surgery, the available data on enhanced recovery remains limited, with no consensus on which components to include within the ERP. The difficulty in identifying specific factors to include within a pathway is in part due to the lack of representative metrics of recovery. Secondly, the strength of ERPs usually lies in the agglomeration of multiple components rather than the individual components themselves. This review provides a brief review on current developments in ERPs in pulmonary surgery, emphasizing novel components in the pre and postoperative care of patients. Furthermore, we discuss the limitations of current metrics used to study recovery, and what steps can be taken to direct future studies that aim to enhance patient recovery after pulmonary surgery.
\end{abstract}

Keywords: Enhanced recovery pathway (ERP); enhanced recovery; pathway; fast-track; pulmonary

Submitted Jun 21, 2018. Accepted for publication Sep 14, 2018.

doi: $10.21037 /$ jtd.2018.09.61

View this article at: http://dx.doi.org/10.21037/jtd.2018.09.61

Surgery implies the concept of "recovery" as a central pillar of the patient trajectory. It has been a focal point of surgical research for ages; however, the specific components that define surgical recovery are not well defined. Historically, measures such as perioperative mortality, morbidity, complications, and hospital length of stay have been used as proxies to define surgical recovery. This disconnect between the definition of recovery and the endpoints we use to measure recovery in research is well addressed by Lee et al. (1) Beyond this, Lee suggests that recovery should be defined as "a rapid decrease in functioning in all relevant domains immediately postsurgery and persistence in this postoperative state during the deterioration period, which will gradually "recover" or exceed the baseline value over the rehabilitation period." (1) (Figure 1).

As such, the concept of recovery begins from the preoperative baseline of the patient, traverses through the immediate deterioration associated with the early postoperative period, and continues with the long-term patient rehabilitation. Addressing and optimizing these various phases of the patient experience has been the fundamental principle in the development of standardized post-operative protocols, so called enhanced recovery pathways (ERPs).

ERPs are not new and surgeons have been trying to optimize patient recovery for decades by standardization of post-operative protocols. However, modern ERPs are characterized by an attempt to break free from 


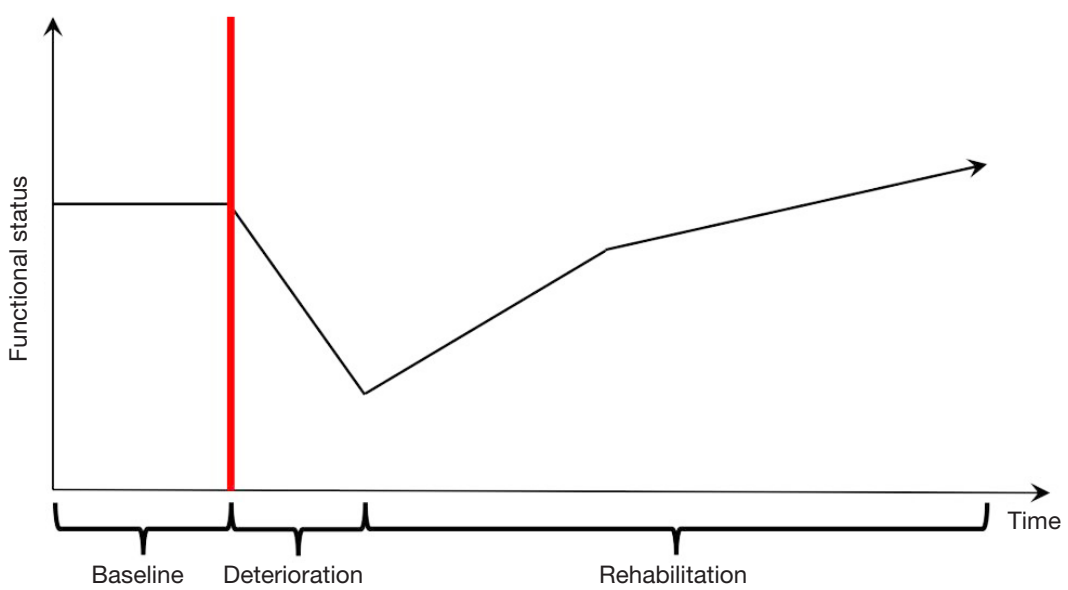

Figure 1 Phases of recovery [modified from Lee et al. (1)].

surgical dogma and tradition. Instead, ERPs aim to standardize patient care using evidence-based methods and encourage multidisciplinarity. While initially focused on the postoperative management, ERPs have now grown to encompass all phases of the patient trajectory, with emphasis on the preoperative, perioperative, and postoperative management of patients. The preoperative phase encompasses initial assessment and surgical planning, optimizing preoperative functional and nutritional status, and managing and setting realistic expectations for recovery, both within the hospital and in the outpatient setting. The intraoperative component is by far the bulk of the literature, as it encompasses surgical approach (minimally invasive $v s$. open), anesthetic approach (multimodal, local anesthesia, general anesthesia), and perioperative management (fluid management, body temperature, etc.). Given that the benefits of minimally invasive surgery have extensively been described in the literature, this review spends little time on the surgical approach in order to focus discussion on the new developments in ERPs, specifically the standardizing of pre and postoperative goals and management. Finally, the postoperative period encompasses both the immediate period in the hospital, and the long-term period of recovery once a patient leaves the hospital. Specifically it targets broad components such as patient mobilization, early return to oral nutrition, pain management, and drain management (2). An example of our postoperative pathway management is provided here (Table 1). Therefore, by targeting all aspects of the operative period, the aim of ERPs should be to return the patient to the best possible functional status in the shortest amount of time. In essence improved postoperative outcomes and early discharge from hospital are by-products of the true goal, which is to minimize the functional impact of surgery.

This concept is particularly relevant in oncologic pulmonary surgery where the goal of the operation is cancer control, a consequence of which is inevitable and permanent loss of respiratory capacity. As such, a "return to baseline" is inherently impossible, which puts into light the lack of adequate metrics for patient-reported outcomes (PROs) and functional status in the postoperative period. The limitations of proxies of recovery such as length of stay or complications rates highlights the need for more holistic metrics of recovery such as PROs or functional outcomes. Within this context, Kim et al. introduced the metric of return to intended oncologic therapy (RIOT) which addressed whether or not a patient "recovered" enough in the postoperative period to be able to tolerate postoperative adjuvant therapy (3). RIOT is a combined metric that included whether or not the patient was able to initiate post-operative therapies, and a time metric for the delay until therapy initiation (3). When measured in the context of ERPs for liver resection, the ERP patients showed lower symptom burden on life interference and overall shorter time interval to return to baseline functional status, which resulted in a more rapid RIOT (4). The concept of PROs such as RIOT is novel and as such only very few studies using ERPs have incorporated them to assess for patient recovery after hospital. Nevertheless, incorporating metrics that can more accurately measure recovery is absolutely necessary for future research around perioperative recovery, especially in the context of ERPs.

The benefits of ERPs have been demonstrated across surgical specialties, with benefits mainly in reducing length 
Table 1 Example of enhanced recovery pathway for lobectomy [modified from Madani et al. (2)]

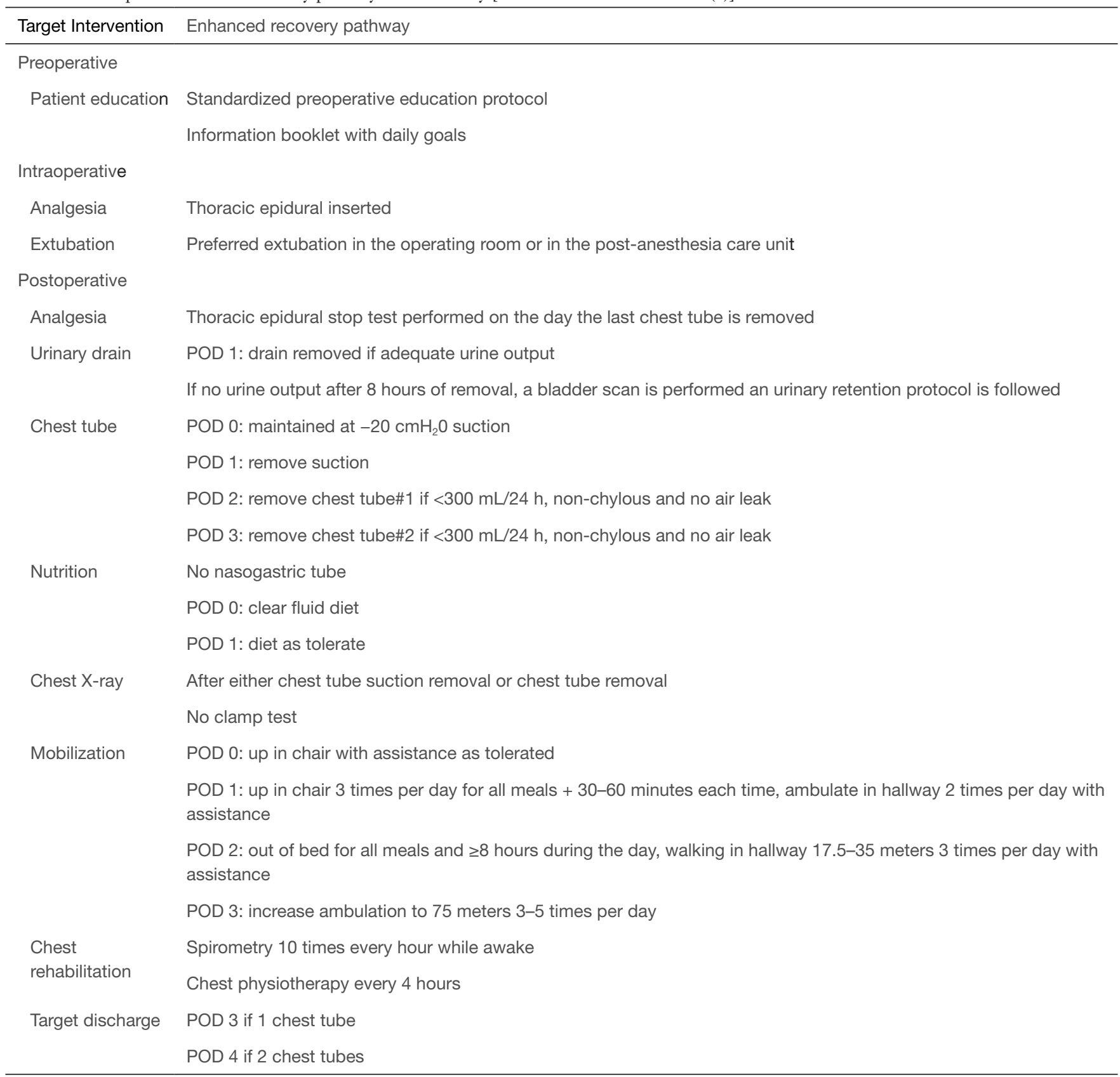

POD, post-operative day.

of hospital stay and overall complication rates. However, the bulk of studies and benefits have been demonstrated in the context of colorectal surgery, where the adoption of ERPs was a significant shift from the conventional historical management (5). In the context of thoracic surgery, specifically with regards to lung resection, the available literature is more limited. Indeed, a systematic review performed by Fiore et al. was the first to synthesize the last decade's worth of studies assessing the benefits of ERPs in pulmonary surgery (6). Interestingly, all nonrandomized studies demonstrated a significant reduction in overall length of stay (6). However, the sole randomized controlled trial by Muehling et al. failed to showed a significant difference in length of stay between the ERP and conventional groups (7). 
As such, some have suggested that the difference in length of stay in nonrandomized studies may be a result of bias in the selection of ERP patients (8). Alternatively, Muehling et al. failed to include a protocol on chest tube management in their ERP, which could have significantly affected their length of stay as other studies have effectively demonstrated that chest tube duration represents a major determinant of LOS in thoracic patients (6). However, what Fiore et al. demonstrate overall, is that when comparing ERP to conventional approach, there is no difference in overall complication rates, readmissions rates, and overall mortality rates (6). These findings together suggest that ERPs in thoracic surgery may reduce overall length of stay without compromising patient care. Furthermore, while the overall complication rates show no significant difference, the rate of short term complications and overall morbidity have been reported to be lower in several studies (6). Indeed, in our centre's experience on 234 patients who underwent elective lung resections, we found an overall 30-day complication rate, largely determined by the very significant reduction in urinary tract infections with early Foley removal as part of the ERP protocol (2). While these findings suggest some benefit in using ERPs in thoracic surgery, the data had several pitfalls. Indeed, due to the wide variability between individual ERP components and the low volume of studies (1 randomized trial and 6 nonrandomized studies), the data was deemed of low quality (6). This variability in results can be attributed to the difficulty of studying individual components of each pathway, as often it is not a single component that defines a benefit but more the agglomeration of several components together (2). Indeed, many of the studies included had key differences in the components of both their standard and ERP protocols, with some standard protocols already including many of the components of the ERPs and thus undermining the effects of the "novel" pathway. Beyond this, the primary outcome was often length-ofstay (LOS), which as we've previously discussed represents an incomplete and indirect measure of recovery at best. The sole RCT published showed no significant difference in length of stay, putting forth the idea that some of the benefit seen in non-randomized studies may be the result of bias. At the time, it was evident that more prospective trials were necessary to make conclusions regarding ERPs. In 2017, Li et al. published a substantial update, this time meta-analyzing the results of 7 RCTs for a total of 486 patients (9). Here they indicated that ERP patients had significant reductions in morbidity, with emphasis on a drop in pulmonary and surgical complications (9). They found no difference in cardiac complications or mortality (9). Their study represents the first meta-analysis to demonstrate significant improvement in perioperative outcomes in the context of ERPs and thoracic surgery. Thus, when taken together, these findings suggest that ERPs have benefit regarding perioperative morbidity, with some improvements in hospital and ICU length of stay. Together however, they remain limited in their ability to measure post-operative recovery given that they lack comprehensive metrics of recovery. As such, further study is still required to assess the effects of ERPs on recovery, with special emphasis on (I) identifying valid metrics of recovery and (II) developing a consensus on what to include within an ERP protocol.

Streamlining the perioperative process itself puts forth the idea that ERPs may have a role in reducing healthcare costs associated to lung resection. However, simply calculating based on the cost of hospital stay represents a limited and incomplete view of the costs of postoperative recovery (10). Based on this, Paci et al. set forth to assess whether ERPs have a significant economic impact by calculating cost during hospitalization (institutional costs), in the immediate follow-up (healthcare system costs), and the cost burden on the patient and caregivers (societal costs) in patients who had undergone ERP vs. conventional pathway in our centre (10). Similar to previous publications, they found a reduced median length of stay $(2,10)$. However, their most striking result was that the ERP group demonstrated a lower caregiver burden (defined as hours spent by a caregiver in assisting the patient postoperatively) concomitant with a lower societal cost, with a mean difference of $\$ 4,396$ less in the ERP group (10). These findings have significant repercussions in our healthcare system, which is publicly funded and as such constrained by limited resources. First, a shorter length of stay represents a more rapid turnover of available beds in the hospital, thus speeding up the process from first visit to surgery in our patient population (10). Secondly, with reduced overall costs, ERPs not only reduce the burden on the public system but on the patients and relatives in the postoperative period $(10,11)$. Finally, on could hypothesize that reduced caregiver burden is an indication that patients have returned to baseline function and autonomy in an accelerated fashion. These findings may in fact demonstrate the strongest impact of ERPs in all surgical contexts.

Based on this, the introduction of new features to ERPs is a hot topic in the literature currently. As previously discussed, ERPs have expanded their breadth to include 
the preoperative phase, the perioperative phase, and the postoperative phase. Implementation of smoking cessation programs preoperatively have long been included in preoperative optimization of lung cancer surgical patients, with positive results although strong data remains limited (12). Most recently however, the implementation of optimizing preoperative functional baselines has garnered interest. In their meta-analysis, Pouwels et al. identified 11 studies, for a total of 277 study participants, that compared the implementation of a preoperative exercise therapy program to the current standard of care (no preoperative program). The individual studies collectively suggested that pre-operative exercise is associated with benefits in the post-operative period, with specific regards to complication rates, mortality, hospital length of stay, post-operative fitness levels and overall quality of life (13). However, due to the heterogeneity of the study populations and the exercise programs themselves, a meta-analysis was not performed, and as such no definite conclusions could be drawn (13). While the data on prehabilitation in pulmonary surgery remains limited, the benefits of preoperative exercise programs in improving postoperative functional exercise capacity have been demonstrated in several other surgical specialties, including other fields within thoracic surgery (14). Indeed, our own center found that prehabilitation in patients undergoing esophago-gastric resections resulted in improved functional status both pre and post-operatively. As these programs likely to become a mainstay in preoperative care, the next step remains to develop standardized definitions of pre-habilitation, paying specific attention to aspects such as which routine to follow, how early before surgery to start a program and at what intensity and frequency to execute such a program (13).

Several other aspects such as preoperative nutrition, specific cardiopulmonary evaluations, optimization of chronic conditions, and a slew of other preoperative factors are undergoing evaluation. With regards to the perioperative period, the use of posterior intercostal nerve blocks as opposed standard thoracic epidural analgesia is an exciting development in the field. A groundbreaking study by Rice $e t a l$. demonstrated that in patients undergoing lung surgery, those who received intercostal nerve blockade as opposed to thoracic epidural analgesia had a significant reduction in mean hospital stay without any difference in postoperative complications, overall pain scores, and narcotic utilization (15). These findings represent a key development as the use of epidural analgesia is associated with side effects such as hypotension, urinary retention, nausea, and vomiting, all of which are potential factors that can delay discharge in thoracic patients (15). Further to this, patients can safely be discharged with intercostal nerve blockade as opposed to epidurals, which required in-hospital management (15). Enhancements in the postoperative management has surfaced in the form of portable chest tube suction devices, the use of specific oxygen regimens in the immediate postoperative period, and a plethora of other novel ideas whose benefits remain to be shown $(16,17)$. All of this taken together exemplifies that ERPs in thoracic surgery are still in their infancy. Indeed, the lack of strong evidence to show benefits of ERPs is likely strongly determined by the significant variability between available protocols in the literature. These protocols themselves will be subject to considerable change as new aspects of perioperative care are discovered and implemented, such as prehabilitation programs, nutritional programs, and others.

It is clear that ERPs are here to stay in modern thoracic surgery management. At the very least, ERPs deliver the same standard of care as conventional approaches. At best, they are associated with reduced length of stay and reduced costs, both on the system and the patient themselves. However, both the specific components and the scope of ERPs are expanding and developing each year. Indeed, ERPs now include the entire operative experience, from preoperative planning to long-term postoperative rehabilitation. As new components surface in the literature, ERPs will undergo major shifts. As they become more comprehensive, one can hope that the demonstrated benefit of ERPs will become more apparent in the overall context of patient recovery after surgery. As new metrics for recovery are developed and implemented into ERP research, the more nuanced effects of ERPs may be identified with regards to overall patient recovery. Finally, it is critical to note the iterative nature of ERPs. Like all aspects of surgery, pulmonary surgery is a constantly evolving entity. ERPs must be revised and revisited over time to reflect this reality. They must provide continuous and incremental improvements to the experience of those patients who entrust their lives to our care.

\section{Acknowledgements}

None.

\section{Footnote}

Conflicts of Interest: The authors have no conflicts of interest 
to declare.

\section{References}

1. Lee L, Tran T, Mayo NE, et al. What does it really mean to "recover" from an operation? Surgery 2014;155:211-6.

2. Madani A, Fiore JF Jr, Wang Y, et al. An enhanced recovery pathway reduces duration of stay and complications after open pulmonary lobectomy. Surgery 2015;158:899-908; discussion 908-10.

3. Kim BJ, Caudle AS, Gottumukkala V, et al. The Impact of Postoperative Complications on a Timely Return to Intended Oncologic Therapy (RIOT): the Role of Enhanced Recovery in the Cancer Journey. Int Anesthesiol Clin 2016;54:e33-46.

4. Day RW, Cleeland CS, Wang XS, et al. Patient-Reported Outcomes Accurately Measure the Value of an Enhanced Recovery Program in Liver Surgery. J Am Coll Surg 2015;221:1023-30.e1-2.

5. Nicholson A, Lowe MC, Parker J, et al. Systematic review and meta-analysis of enhanced recovery programmes in surgical patients. Br J Surg 2014;101:172-88.

6. Fiore JF Jr, Bejjani J, Conrad K, et al. Systematic review of the influence of enhanced recovery pathways in elective lung resection. J Thorac Cardiovasc Surg 2016;151:708-15.e6.

7. Muehling BM, Halter GL, Schelzig H, et al. Reduction of postoperative pulmonary complications after lung surgery using a fast track clinical pathway. Eur J Cardiothorac Surg 2008;34:174-80.

8. Brown LM. "Moving right along" after lung resection, but the data suggest "not so fast". J Thorac Cardiovasc Surg

Cite this article as: Eustache J, Ferri LE, Feldman LS, Lee L, Spicer JD. Enhanced recovery after pulmonary surgery. J Thorac Dis 2018;10(Suppl 32):S3755-S3760. doi: 10.21037/ jtd.2018.09.61
2016;151:715-6.

9. Li S, Zhou K, Che G, et al. Enhanced recovery programs in lung cancer surgery: systematic review and metaanalysis of randomized controlled trials. Cancer Manag Res 2017;9:657-70.

10. Paci P, Madani A, Lee L, et al. Economic Impact of an Enhanced Recovery Pathway for Lung Resection. Ann Thorac Surg 2017;104:950-7.

11. Kumar R, Donahue JM. Editorial for economic impact of an enhanced recovery pathway for lung resection. J Thorac Dis 2018;10:7-9.

12. Schmidt-Hansen M, Page R, Hasler E. The effect of preoperative smoking cessation or preoperative pulmonary rehabilitation on outcomes after lung cancer surgery: a systematic review. Clin Lung Cancer 2013;14:96-102.

13. Pouwels S, Fiddelaers J, Teijink JA, et al. Preoperative exercise therapy in lung surgery patients: A systematic review. Respir Med 2015;109:1495-504.

14. Minnella EM, Carli F. Prehabilitation and functional recovery for colorectal cancer patients. Eur J Surg Oncol 2018;44:919-26.

15. Rice DC, Cata JP, Mena GE, et al. Posterior Intercostal Nerve Block With Liposomal Bupivacaine: An Alternative to Thoracic Epidural Analgesia. Ann Thorac Surg 2015;99:1953-60.

16. Ansari BM, Hogan MP, Collier TJ, et al. A Randomized Controlled Trial of High-Flow Nasal Oxygen (Optiflow) as Part of an Enhanced Recovery Program After Lung Resection Surgery. Ann Thorac Surg 2016;101:459-64.

17. George RS, Papagiannopoulos K. Advances in chest drain management in thoracic disease. J Thorac Dis 2016;8:S55-64. 